\title{
Correction to: Remarks on Hierarchic Control for a Linearized Micropolar Fluids System in Moving Domains
}

\section{Isaías Pereira de Jesus ${ }^{1}$}

Published online: 18 September 2018

(c) Springer Science+Business Media, LLC, part of Springer Nature 2018

\section{Correction to: Appl Math Optim (2015) 72:493-521 https://doi.org/10.1007/s00245-015-9288-2}

The original version of this article unfortunately contained a mistake in the equation.

On page 516, Equation there should be

$$
\begin{gathered}
\inf _{(\boldsymbol{\xi}, \eta)}\left\{\frac{1}{2} \int_{\mathcal{O} \times(0, T)}|\operatorname{det} K(t)|\left(|\varphi|^{2}+|\psi|^{2}\right) d y d t+\epsilon\|(\boldsymbol{\xi}, \eta)\|_{\mathbf{L}^{2}(\Omega) \times L^{2}(\Omega)}\right. \\
\left.\quad-\left((\xi, \eta),\left(z^{T}, w^{T}\right)\right)_{\mathbf{L}^{2}(\Omega) \times L^{2}(\Omega)}\right\}
\end{gathered}
$$

instead of

$$
\begin{gathered}
7 \inf _{(\xi, \eta)}\left\{\frac{1}{2} \int_{\mathcal{O} \times(0, T)}|\operatorname{det} K(t)|\left(|\varphi|^{2}+|\psi|^{2}\right) d y d t+\epsilon\|(\boldsymbol{\xi}, \eta)\|_{\mathbf{L}^{2}(\Omega) \times L^{2}(\Omega)}\right. \\
\left.-\left((\xi, \eta),\left(z^{T}, w^{T}\right)\right)_{\mathbf{L}^{2}(\Omega) \times L^{2}(\Omega)}\right\}
\end{gathered}
$$

The original article can be found online at https://doi.org/10.1007/s00245-015-9288-2.

$凶 \quad$ Isaías Pereira de Jesus

isaias@ufpi.edu.br

1 Dpto. Matemática, Universidade Federal do Piauí, Campus Ministro Petrônio Portela - Ininga, Teresina, PI 64049-550, Brazil 